\title{
能耗、大气污染物排放及碳排放协同研究可视化分析
}

\author{
李红梅 \\ 新疆中汇新能环保科技有限公司石河子分公司 \\ DOI:10.12238/eep.v3i8.953
}

\begin{abstract}
[摘 要] 现阶段, 越来越多的人开始关注生态环境问题。为进一步提高能耗、大气污染排放物及碳排放 协同研究的可视化水平, 在知名数据库中, 对与上述指标内容相关的数据进行搜集, 对其协同性研究的 文献记录加以归纳, 采用计量软件Citespace对参考文献展开可视化分析, 可得到协同研究的知识基础 和热点演变等成果。
\end{abstract}

[关键词] 能耗; 大气污染物; 碳排放

中图分类号: F832. 5 文献标识码: A

近年来, 城市化进程明显加快。与此 同时, 我国的能源与资源消耗量不断增 加。虽然经济实现了快速增长, 但是大气 污染、碳排放超标问题也日益突出。目 前, 我国已经将国策更新为保护环境、节 约资源, 致力于构建环境友好型的社会。 基于此, 学术领域中越来越多的学者开 始关注能耗问题、大气污染物问题、碳 排放问题。因而, 本文从文献梳理的角度 上, 对协同研究的可视化展开分析, 具有 现实性研究价值与意义。

\section{1 数据来源与研究工具}

1. 1 数据来源

研究中所选取的可视化分析数据 源, 以Web of Science核心合集为主。 具体的数据检索方法为: 首先, 高级检 索。在进入到高级检索中后, 要依据实 际情况合理设置检索条件。其中, 以

“ALL lan-guage”、“ALL document types”为主, 并不对检索结果进行限制。 其次, 合理选择数据。在对数据进行选择 时, 要围绕时间的跨度, 对所有年份的文 献进行剖析, 确保数据来源的全面性与 广泛性。最后, 确定索引源并保存选中文 献。通过所确定的索引源如 IC、CPCI-S 等, 共计检索出 280 余条记录, 以 $t x t$ 格式 保存所有的检索结果。就统计结果来 看, 2001年最早出现关于能耗、大气污染 物和碳排放的相关报道。Warren CD在研 究中, 认为大气污染物主要是汽车燃油
造成的, 使美国空气产生了污染性的负 担。因此, 工业界和政府部门要共同协 作, 尽量减少对汽车尾气的排放。从 2012年开始至今, 已经有越来越多关于 能耗、大气污染物、碳排放的相关发文, 且从整体上看来始终保持上升的趋势。 可以表明, 学术领域中的学者比较关注 协同性研究。

\section{2 研究工具}

本次研究中, 选择的研究工具, 是一 种引文性的分析软件。CiteSpace (引文 空间) 作为分析软件, 其重点是对蕴藏的 潜在性知识进行深入分析, 以可视化的 方式, 将科学的知识结构、知识规律、知 识分布等, 有效呈现出来。该分析软件的 研发者, 是以陈超美博士为代表的团队, 研发背景和平台是Java。对该分析软件 的作用分析, 发现其不仅具有较为强大 的功能, 能够从合理的角度上对各步骤 处理, 同时也能够显示出重点内容或是 核心内容。目前, 该分析软件已经被广泛 应用于科学知识的研究领域中, 当作是 可视化图谱绘制的工具。

\section{2 研究成果}

2.1协同研究的知识基础

在研究中, 于CiteSpace软件中, 导入 采集到的题录, 并设置时间范围最早为 2001 年, 最晚为 2008 年, 以 1 年为周期。即 节点类型, 选择的是 “Cited Reference”, 不改变其他选项。由此, 获得节点 $\mathrm{N}$, 数值
为 294 ; 连线 $\mathrm{E}$, 数值为 699 。文献被引用 的次数, 以节点进行表示。也就是说, 节 点越小, 说明文献被引用的次数越少; 反 之, 被引用的次数越多。此外, 文献地位 的衡量指标, 也可用节点的突现性、中心 性加以表示。突现性、中心性高, 说明文 献在领域中的作用突出。在产生研究报 告后, 可通过被引用的频率、突现性、中 心性等, 归纳出关键文献。梳理关键性文 献, 发现文献发表的最早时间是2007年。 其中, 多数文献集中于 2009 年, 此种现象 说明不少学者在三者协同研究中都有成 果, 只不过研究深入度不足。就研究的内 容而言, 归纳出的关键文献更倾向于亚 洲地区的中国。比如, OHARA T (2007) 在 其研究中, 结合历史、现在与未来的碳排 放量, 从科学的角度上为亚洲制定了关 于1980年至2020年的碳排放清单。且研 究认为, 碳排放量会随着能源消耗量的 增加, 而不断增长, 中国的碳排放量会在 极大程度上影响亚洲总排放量。ZHANG Q (2009) 依据亚洲生态环境的现状, 制 定出新的空气污染物排放清单。在排放 清单中, 对所有人为性造成的碳排放量 进行预估, 并对中国的排放量进行突出 强调。为更好的统计中国能源, 掌握中 国碳排放情况, 对中国的能源和碳排放 展开详细的审查, 对二氧化碳和一氧化 氮等大气污染物、碳排放量进行预测, 且预测的结果是将呈现出持续上升的 
趋势。KLIMONT Z (2009) 在研究中提到, 亚洲二氧化硫的增长速度, 将会出现趋 于稳定的下降现象, 氮氧化合物排放量 在一段时间内将会上升, 最后上升的幅 度将会趋于缓慢。随着然料使用量的降 低, 碳排放含量也将会呈现出下降的趋 势, 不过碳排放量与上述两种物质相比, 其估计值仍存在很大的不确定。

\section{2 协同研究的热点演变}

在对文献进行检索与分析中, 关键 词是对文献核心内容的高度概括。所以, 借助CiteSpace软件, 可深入分析关键 词, 从关键词较多获取研究领域的热点, 从研究年份角度展现出不同时期的研 究热点。在CiteSpace中, 节点类型以

“Keywords”为主, 其他选项与协同研究 知识基础相一致。为了避免热点受检索 词的干扰和影响, 要将与能耗、大气污染 物、碳排放等相类似的检索词汇排除掉。 梳理文献, 选取2001年至2008年期间, 排 名频次在前五的关键词。关键词的节点 大, 说明该关键词在文献中出现的频率 多, 作用也比较突出, 可将其看作是该领 域的研究热点。在不同时期内, 能耗、大 气污染与碳排放的协同研究热点, 存在 明显的差异。在2001年至2010年期间: 研究初期, 热点并不十分明确。经过较长 时间的探索, 出现词汇为“系统”。SZARKA $N$ (2008) 在研究中, 对系统动力学的模型 加以构建, 该模型主要是起到模拟的作 用, 模拟的内容是能源使用后对空气污 染物排放的影响。CHAVEZ R H(2008) 分 析了新型耦合系统。其在研究中发现, 该系统与现阶段生产的系统进行比较, 其节能效果能达到 $39 \%$, 无论是污染物的 排放量, 还是颗粒物的排放量, 均有所下 降。在2011年至2014年期间: 研究中期,
频率出现最高的词汇是 “中国”。所以, 多数学者将中国作为研究重点。DONG L (2013) 结合能源消耗、产业共生及 HPIMO模型, 对与产业共生的相关情景展 开了综合的分析。并指出, 产业共生能够 在一定程度上减少固体废物的排放, 可 提高能源的使用效率。在 2015 年至 2018 年: 研究末期, 频率出现最高的词汇是 “投入-产出”、“效率”。WANG Y (2017) 以假设提取法展开分析, 其通过对 2010 年中国投入-产出及其排放数据的总结, 以流量图的形式体现了中国空气污染 物的排放量, 发现不同区域和不同行业 的大气污染排放、碳排放不同, 并针对 实际情况制定出合理的控制空气污染 的对策。

\section{3 研究结论}

本文章在研究中, 以Citespace作为 研究工具, 将Web of Science核心合集中, 与能耗、大气污染物、碳排放相关的文 献进行检索, 得出如下结论:

其一, 学者关于协同性研究的起步 时间, 虽然较晚, 不过研究发展的速度较 快。能够证实, 社会各界已经开始认识到 生态环境的重要性, 且对理论进行完善, 能够在环境保护方面发挥作用。

其二, 协同研究知识基础与热点演 变相关文献发表年份, 介于 2001 年至 2018年之间。而且, 研究对象多集中于以 中国为主的三者协同研究方面。能够证 实, 中国在生态环境保护方面有较大的 发展空间, 可展开深入探讨。

\section{[参考文献]}

[1]WARREN C D.Carbon Fiber in Future Vehicles[J].Sampe Jour-na1,2001, 37(2):7-15.

[2]李杰.CiteSpace中文指南[EB/OL]. http://blog.sciencenet.cn/blog-496649 -886962.htm1.

[3]OHARA $\mathrm{T}$,AKIMOTO $\mathrm{H}$,KUROKAWA $\mathrm{J}$,et al.An Asian E-mission Inventory of Anthropogenic Emission Sources for thePeriod 1980-2020[J].Atmospheric Chemistry \& Physics ,2007,7(3):6843-6902.

[4]ZHANG Q ,STREETS D G ,CARMICHAEL $G R$,et al.AsianEmissions in 2006 for the NASA INTEX-B mission[J].Atmospheric Chemistry and Physics Discussions, 2009,9(1):5131-5153.

[5]KLIMONT Z,COFALA J,XING J,et a1.Projections of SO2,NOx,and Carbonaceous Aerosols Emissions in Asia[J].Tellus SeriesB-chemical \& Physical Meteorology, 2009,61 (4):602-617.

[6]SZARKA N,WOLFBAUER K J,BEZAMA A.Atmospheric e-missions modeling of energetic biomass alternatives using sys-tem dynamics approach[J].Atmospheric Environment ,2008,42(3):403-414.

[7]CHAVEZ R H.Pollution Reduction and Energy Savings of a Coupled Two -kiln System for Bricks Making [J]. Environ-mentalProgress ,2008 ,27(3):3 97-404.

[8]DONG L ,FUJITA $T$,ZHANG $H$,et a).Promoting Low-carbon City Through Industrial Symbiosis : A case in China by Ap-plying HPIMO Mode][J].Energy Policy ,2013,61:864-873.

[9]WANG Y,LAI N,MAO G,et a].Air Pollutant Emissions from Economic Sectors in China: A Linkage Analysis [J].Ecologi-calindicators ,2017,77:25 0-260. 